\title{
Current status of nosocomial COVID-19 cases in delivery facilities in Japan: Case-control study
}

\author{
JUNICHI HASEGAWA ${ }^{1}$, Tatsuya Arakaki ${ }^{2}$, Akihiko Sekizawa ${ }^{3}$, Tomoaki Ikeda ${ }^{4}$, Isamu \\ Ishiwata $^{5}$, and Katsuyuki Kinoshita ${ }^{6}$ \\ ${ }^{1}$ Sei Marianna Ika Daigaku \\ ${ }^{2}$ Showa Daigaku \\ ${ }^{3}$ Showa University School of Medicine \\ ${ }^{4}$ Mie University Graduate School of Medicine Faculty of Medicine \\ ${ }^{5}$ Ishiwata Obstetrics and Gynecology \\ ${ }^{6}$ Seijo Kinoshita Hospital
}

September 16, 2020

\begin{abstract}
Objective: To identify the characteristics of and measures taken by Japanese medical facilities providing maternity services that have recorded cases of nosocomial infection with SARS-CoV-2, which causes coronavirus disease 2019 (COVID-19). Design: A nationwide questionnaire survey: case-control study. Setting: Medical facilities providing maternity service in Japan. Population: 1,418 medical facilities providing maternity service (65\% of all delivery facilities in Japan). Methods: A nationwide questionnaire survey about nosocomial COVID-19 was conducted in July 2020. A case-control study was conducted by including medical facilities providing maternity service with (Cases) and without (Control) nosocomial COVID-19. Main outcome measures: Contributed characteristics and variables associated with nosocomial COVID-19. The number of treated pregnant women with COVID-19 until June 2020 and cases of nosocomial COVID-19 in Japan. Results: Seventy-five COVID-19-positive pregnant women were treated in 48 facilities. Responses from Nosocomial infection was reported in $4.1 \%$ of the facilities. Nine reports developed a nosocomial infection in the maternity ward or obstetric department. Variables that contributed to nosocomial COVID-19 were chest CT on admission (adjusted odds ratio [95\% confidence interval], 4.76 [2.44-9.27]), PCR test for SARS-CoV-2 before delivery (2.27 [1.21-4.25]), transfer of pregnant women with fever to another hospital (0.21 [0.06-0.71]), and private clinics $(0.17$ [0.05-0.60]). Conclusion: Nosocomial infection is likely to occur in large hospitals that treat a higher number of patients than private clinics do. Nosocomial infection can occur even in medical facilities performing COVID-19 screening tests, such as chest CT and PCR test.
\end{abstract}

\section{Introduction}

The World Health Organization (WHO) declared the Coronavirus disease 2019 (COVID-19) outbreak a public health emergency of international concern (PHEIC) in January 2020 and a pandemic in March $2020^{1}$. In Japan, since the first case of COVID-19 reported on January 16, $2020^{2}$, the number of domestic infections and deaths have reached over forty-thousand and one thousand, respectively, in August 2020 (Figure 1) .

After declaring the COVID-19 pandemic in March, PCR tests for SARS-CoV-2 for examining symptomatic patients and those in close contact with COVID-19-positive individuals were established as a priority. A state of emergency for COVID-19 had also been declared in highly populated prefectures on $7^{\text {th }}$ April 2020. In these areas, the government closed schools, companies, and shops except facilities related to life lines, and requested citizens to stay home. Consequently, the COVID-19 pandemic in Japan was under control until the cancellation of this declaration on $25^{\text {th }}$ June. 
The UK Obstetric Surveillance System (UKOSS) demonstrated an estimated incidence of 4.9 COVID-19 cases during pregnancy per 1000 maternities $^{3}$. Despite the increasing number of published studies on COVID-19 in pregnancy, there is currently limited data on the perinatal outcomes of the infection.

A report from a hospital in Wuhan suspected that $41 \%$ of patients, and $26 \%$ of ICU-patients possibly had hospital-related transmission of COVID-19 ${ }^{4}$. Since COVID-19 can transmit person-to-person and a significant number of carriers are asymptomatic due to low virulence and longer incubation period of the virus, asymptomatic carriers and patients in the incubation period, including health care workers, did not take adequate precautions and thus, became a source of transmission ${ }^{4-6}$.

In Japan, the maternity service system is slightly different from that in other developed countries. There are more than 2000 maternity facilities that provide delivery services throughout Japan for approximately 860 thousand deliveries per year. More than half of all deliveries are managed in private clinics operated by one or two obstetricians, since pregnant women are more likely to prioritize accessibility and comfort of such a private delivery facility. In these clinics, Polymerase Chain Reactions (PCR) tests, chest computed tomography (CT), and detailed laboratory tests to screen for COVID-19 are usually not available.

Therefore, the Japan Association of Obstetricians and Gynecologists (JAOG) planned a nationwide survey related to COVID-19 cases in medical facilities providing maternity services to identify the characteristics and measures implemented by facilities that have recorded cases of nosocomial COVID-19.

\section{Methods}

\section{Study design and participants}

The nationwide questionnaire survey was conducted in July 2020 as an investigation by the JAOG. The questionnaire was sent to all medical facilities providing maternity services in Japan. The questionnaire, accompanied by a cover letter outlining the aims of the study, was sent via post to the director, chief obstetrician, or consultant of the feto-maternal medicine or delivery facilities. The answers to the questionnaire were thereafter received via the web form.

The questionnaire included questions regarding the number of deliveries in each facility in 2019, the number of treated pregnant women with COVID-19 until June 2020, cases of nosocomial COVID-19, measures during daily clinical practice for general outpatients, measures during daily clinical practice for inpatients whose admission was not due to an infectious disease, and measures during labor for parturients without confirmed or suspected COVID-19.

The subjects were divided into two groups: medical facilities that had recorded cases of nosocomial COVID19 and control facilities that did not record any cases of nosocomial COVID-19. A case-control study was conducted to compare variables associated with clinical characteristics, risk factors, and measures against COVID-19.

\section{Statistical analysis}

The data were entered into a computerized data analysis program (Statistical Package for Social Science (SPSS), Windows version 25.0J; Chicago, IL, USA). Categorical variables were reported as percentages and compared using Fisher's exact test. The multivariable analyses were based on logistic regression analyses. Significant contributing factors determined by the univariate analyses between medical facilities that experienced nosocomial infection and controls were used in the multivariable analyses. Statistical significance was defined as a P-value of less than 0.05 .

\section{Role of the funding source}

The funder had no role in study design, data collection, data analysis, data interpretation, or writing of the report. The corresponding author had full access to all data in the study and had final responsibility for the decision to submit for publication.

Patient and public involvement 
Our study was conducted in response to public concerns about nosocomial infection associated with COVID19 infection. The outcomes in our study will be informed by public and patient anecdotal information via statements from JAOG.

\section{Results}

The questionnaire was sent to 2,185 facilities, and responses from 1,418 facilities were assessed (65\% of all delivery facilities). Based on the answers, the total number of deliveries in 2019 among the facilities was 611,444 (71.1\% of total deliveries in Japan). Of these, seventy-five COVID-19-positive pregnant women were treated in 48 facilities.

Nosocomial infections in delivery facilities were reported in $4.1 \%(58 / 1418)$ of the facilities. In prefectures that had declared a state of emergency for COVID-19, 7.2\% (39/541) of facilities had reported cases of nosocomial infections. In other prefectures, which did not declare a state of emergency, $2.2 \%$ (19/858) of facilities had nosocomial infections.

The study flow diagram is shown in Figure 2. Nosocomial infection occurred in fifty of the 652 medical facilities that could treat pregnant women who were confirmed or suspected to be COVID-19 positive (7.7\% of treatable facilities), while 8 cases occurred among 766 medical facilities that could not treat COVID19 positive pregnant women $(1.0 \%$ of untreatable facilities) $(\mathrm{p}<0.01)$. Nine cases $(0.6 \%$ of all facilities) were nosocomial infections in the maternity or in the obstetric departments. Four patients experienced a nosocomial infection in the inpatient ward, two cases in the maternity ward, two in the medical office, and one in the obstetric outpatient clinic. The infection route through medical personnel was estimated in six cases $(66 \%)$, whereas infection route through COVID-19-positive patients was reported in only one case. After the occurrence of a nosocomial infection, delivery service was interrupted in 5 facilities and the outpatient clinic was interrupted in two facilities. It took more than two weeks to recover normal practice in these facilities.

Characteristics and parameters for patients from all the assessed medical facilities is shown in Table $\mathbf{1}$, and the results of multivariate logistic regression analysis are shown in Table $\mathbf{2}$. Variables that contributed to nosocomial infection of COVID-19 (adjusted odds ratio [95\% confidence interval]) were found to be chest CT on admission (4.76 [2.44-9.27]), PCR test for SARS-CoV-2 before delivery (2.27 [1.21-4.25]), maternal transfer of pregnant women with fever to tertiary hospital $(0.21[0.06 .0 .71])$, and private clinic $(0.17[0.05 .0 .60])$.

\section{Discussion}

\section{Main findings}

Nosocomial infection was reported in $4.1 \%$ of medical facilities providing maternity services in Japan. Of these, $58 \%$ occurred in the perinatal center, while only $5 \%$ occurred in maternity private clinics, which are likely to reject medical practices required for symptomatic or suspected COVID-19 cases. Nosocomial infections occur more frequently in tertiary hospitals, which treat many high-risk COVID-19-positive patients.

\section{Strengths and limitations}

Results of the present study showed the current status of nosocomial infection of COVID-19 in delivery facilities in Japan and is the first important report to formulate preventive measures for future nosocomial infections of COVID-19. The limitation of the present study was that the analysis was based on a questionnaire survey that asked for experiences and parameters associated with COVID-19. Causal evaluations between variables and actual nosocomial infections are limited.

\section{Interpretation}

In the maternity ward, person-to-person transmission of COVID-19 was not associated to contact during labor due to the use of personal precaution equipment, including patient's face mask, medical gown, surgical face mask, and goggle or face shield, regardless of whether medical facilities had experienced nosocomial infections. Strangely enough, limited attendance of birth partners during labor was seen more in medical 
facilities that reported nosocomial infection rather than in those which did not experience nosocomial infections. A small number of studies have shown that viral isolates were absent in the amniotic fluid, cord blood, breast milk, and neonatal throat swabs in a subset of patients with COVID-197. So far, standard precautions seem to be effective in labor and delivery rooms for low-risk parturients.

Nosocomial infection in the maternity ward or obstetric department occurred in 9 facilities, and two-thirds of them were associated with the attending medical staff. This suggests that nosocomial infections of COVID-19 are more likely to originate from asymptomatic patients or medical staff rather than through patients confirmed to be positive for COVID-19. In fact, goggles or face shields were used only in $30 \%$ of the inpatients wards and outpatient clinics, though surgical face masks were frequently used. Nosocomial infection associated with COVID-19 might be characterized by unintentional transmission through health care workers from asymptomatic carriers in large hospitals. This tendency is similar to reports from Wuhan, in which asymptomatic carriers and patients did not take adequate precautions and became a source of transmission; consequently, these affected cases included health care workers ${ }^{4-6}$.

Interestingly, typical screening tests for COVID-19, like PCR test for SARS-CoV-2 and chest CT, were frequently performed on admission or before delivery in medical facilities that reported cases of nosocomial infection. This is considered as a confounding factor in large hospitals because these examinations are not available in private clinics. However, multivariate regression analysis revealed that these examinations are independent variables that can increase chances of nosocomial infection. We speculate that overreliance among healthcare workers on negative PCR results in most of the examined patients is weakening the daily prevention measures against infection, despite the low accuracy and sensitivity of the PCR. We found that healthcare workers were reassured that PCR and CT-based hospital strategies aided in isolating infected patients into the emergency ward and, albeit slightly, alcohol sanitation in these hospitals is less frequently used both in outpatient and inpatient wards.

We also believe that effective separation of COVID-19 patients from non-infected cases would be the key to success in the prevention of cross-infection ${ }^{8}$. Segregation of medical facilities to isolate symptomatic or suspected COVID-19-positive patients, concomitant with patient's triage can be a safe and effective way to avoid nosocomial infections. A previous study suggested increasing the use of PCR tests, for SARS-CoV2 , coupled with temporary segregation of high-risk cases into a transitional ward until results are obtained might facilitate the triaging process ${ }^{8}$. We agree with this strategy, but additionally we suggest that healthcare workers should not overtly rely on the PCR results.

\section{Conclusion}

Nosocomial infections of COVID-19 is likely to occur in large hospitals, which treat a large number of patients, compared to that in private clinics, regardless of the methods used. Nosocomial infection can occur even in medical facilities performing screening tests for COVID-19, like chest CT and PCR tests. Daily screening of symptomatic patients and strengthening standard precaution is important to prevent nosocomial infection due to COVID-19.

\section{Acknowledgements}

We are grateful to doctors who answered the present questionnaire survey.

Members of department of medical safety, the Japan Association of Obstetricians and Gynecologists (JAOG): Tomoaki Ikeda (Chair), Koyo Yoshida, Isamu Ishiwata, Akihiko Sekizawa, Yasushi Kuribayashi, Shunji Suzuki, Kazuhiko Ochiai, Hirokatsu Kitai, Kentaro Shimura, Kaoru Kimura, Ken Ishitani, Junichi Hasegawa, Masaji Nagaishi, Tatsuya Arakaki, Gen Ishikawa, Susumu Oka, Osamu Samura, Hiroaki Tanaka, Hiroshi Ishikawa, Takao Kobayashi, Yasumasa Ohno, Kayo Osada, Yukiko Kawana, Hiroyuki Seki, Masahiko Nakata, Koji Hashii, Satoshi Hayashi, Takeshi Murakoshi.

President of JAOG: Katsuyuki Kinoshita

\section{Footnotes}


Conflict of interest: The authors declare no competing interests.

Contributors: JH, TA, and AS conceived the study. JH, TA, and AS drafted the initial protocol, analyzed the data, and wrote the first draft of the manuscript. All authors collected data. JH, TA, AS, and II coordinated the study. TA, and AS developed the database and analyzed the data with JH. All authors contributed to drafting of the manuscript. II, TI, and KK are the guarantors for the study. All authors had full access to all of the data in the study and take responsibility for the integrity of the data and the accuracy of the data analysis.

Funding: Nationwide survey associated with COVID-19 infection, The Japan Association of Obstetricians and Gynecologists (JAOG).

Competing interests: All authors have completed the ICMJE uniform disclosure form atwww.icmje.org/coi_disclosure.pdfand declare: No financial relationships with any organizations that might have an interest in the submitted work in the previous three years; no other relationships or activities that could appear to have influenced the submitted work.

Ethical approval: This study was approved by the JAOG (No. 80, July. 1st, 2020). This investigation was conducted according to the principles of the Declaration of Helsinki. Informed consent was not obtained from patients or their families because this study was based on the analysis of institutional forms, and the patient records/information was anonymized prior to the analysis.

Data sharing: Data available on request due to privacy/ethical restrictions

\section{References}

1 (WHO) WHO. WHO Director-General's opening remarks at the media briefing on COVID-19 - 11 March 2020. 2020 [cited; Available from:https://www.who.int/dg/speeches/detail/who-director-general-s-openingremarks-at-the-media-briefing-on-covid-19-11-march-2020

2 Watanabe M. The COVID-19 Pandemic in Japan. Surg Today. 2020;50:787-93.

3 Knight M, Bunch K, Vousden N, Morris E, Simpson N, Gale C, et al. Characteristics and outcomes of pregnant women hospitalised with confirmed SARS-CoV-2 infection in the UK: a national cohort study using the UK Obstetric Surveillance System (UKOSS) UKOSS. 2020.

4 Wang D, Hu B, Hu C, Zhu F, Liu X, Zhang J, et al. Clinical Characteristics of 138 Hospitalized Patients With 2019 Novel Coronavirus-Infected Pneumonia in Wuhan, China. JAMA : the journal of the American Medical Association. 2020.

5 Li Q, Guan X, Wu P, Wang X, Zhou L, Tong Y, et al. Early Transmission Dynamics in Wuhan, China, of Novel Coronavirus-Infected Pneumonia. The New England journal of medicine. 2020;382:1199-207.

6 Lai X, Wang M, Qin C, Tan L, Ran L, Chen D, et al. Coronavirus Disease 2019 (COVID-2019) Infection Among Health Care Workers and Implications for Prevention Measures in a Tertiary Hospital in Wuhan, China. JAMA Netw Open. 2020;3:e209666.

7 Chen H, Guo J, Wang C, Luo F, Yu X, Zhang W, et al. Clinical characteristics and intrauterine vertical transmission potential of COVID-19 infection in nine pregnant women: a retrospective review of medical records. Lancet. 2020;395:809-15.

$8 \mathrm{Ng}$ PC. Infection Control Measures for COVID-19 in the Labour Suite and Neonatal Unit. Neonatology. 2020;117:141-3.

Figure legend

Figure 1: Cumulative number of patients and deaths due to COVID-19 in Japan

Figure 1: Study flow diagram 
Table 1. Characteristics and parameters for patients from medical facilities with and without cases of nosocomial infection in Japan

Nosocomial infection

Characteristics of institution

Measures during daily clinical practice for outpatients

Measures during daily clinical practice for inpatients

Measures during labor for parturient without confirmed or suspected COVID-19

\section{Nosocomial infection}

Characteristics of institu

Perinatal center

Private clinic

Number of obstetricians wo

Number of delivery (2019)

Never treat patients with $\mathrm{C}$

Maternal transfer to other $\mathrm{b}$

in pregnant women had a fe

in pregnant women with fev

in pregnant women confirme

Measures during daily c

Measurement body tempera

Confirmation of symptoms

Alcohol disinfection (patient

Face mask (patients)

Medical gown

Surgical face mask

Goggle or face shield

Reduction of booking

Limited attendance

Interruption of mothers clas

Telemedicine

Measures during daily c

Measurement body tempera

Confirmation of symptoms

Alcohol disinfection (patient

Face mask (patients)

Medical gown

Surgical face mask

Goggle or face shield

PCR test for SARS-CoV-2

Antigen test for SARS-CoV

Antibody test for SARS-Co

Chest CT

Limited attendance

Measures during labor $\mathbf{f}$

Face mask (patients)

Medical gown

Surgical face mask

Goggle or face shield

PCR test for SARS-CoV-2

Antigen test for SARS-CoV

Antibody test for SARS-Co

Limited attendance

Data indicate $\%$ and $n$, or $n$ 
Table 2. Variables contributing to nosocomial infection of COVID-19 - result of multivariate logistic regression analysis

Variables

Chest CT on admission

PCR test for SARS-CoV-2 before delivery

Number of obstetricians working in institution

Maternal transfer of pregnant women with fever to tertiary hospital

Private clinic
Chest CT on admission

PCR test for SARS-CoV-2 before de

Number of obstetricians working in i

Maternal transfer of pregnant wome

Private clinic

Figure 1: Cumulative number of patients and deaths due to COVID-19 in Japan



Figure 2: Study flow diagram

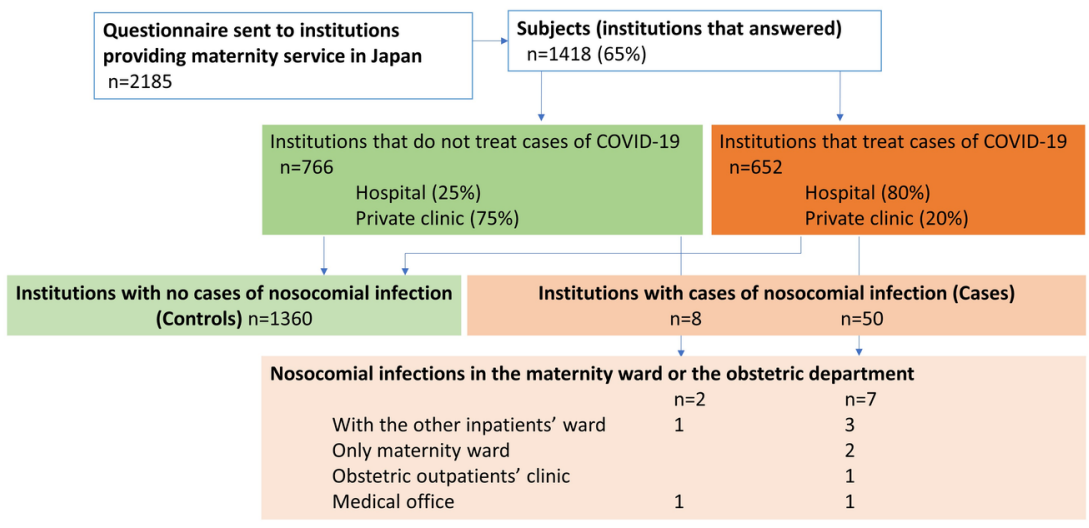

